\title{
New Insight on Hubble 4
}

\author{
Carlos R. Rabaça ${ }^{(1)}$, François Cuisinier ${ }^{(1)}$, Silvia Lorenz-Martins ${ }^{(1)}$, \\ Daniel N. Epitácio Pereira ${ }^{(1)}$, Denise R. Gonçalves ${ }^{(2)}$, Erwan \\ Lastennet ${ }^{(1)}$ \\ (1) Departamento de Astronomia - UFRJ, Ladeira do Pedro Antonio 43, \\ 20080-090 Rio de Janeiro, Brazil \\ ${ }^{(2)}$ Instituto de Astrofísica de Canarias, Spain
}

\section{Introduction}

We started a program of identification of faint structures in PNe on HST archive images, based on the "à trous" wavelet transform (Starck et al. 1998). Using this technique, an image is decomposed in a new set of $\mathrm{N}$ images, each with the same number of pixels as the original one, but containing only the structures of a characteristic scale size (going from one image to the next, the characteristic scale vary by a factor of two). The original image $c_{0}$ can be expressed as the sum of all wavelet scales plus a residual smoothed array $c_{N}$ by $C_{0}(x, y)=C_{N}(x, y)+$ $\sum_{j=1}^{N} w_{j}(x, y)$.

\section{The Wavelet Transform Applied to Hubble 4}

Hubble 4 is a well studied bipolar planetary nebula (e.g., Gonçalves et al. 2001; Hajian et al. 1997; López et al. 1997). It presents a FLIERs that have the peculiarity of being off-axis ( $62^{\circ}$ from the main bipolar axis).

We retreived the four WFPC2 frames available in the $\mathrm{F} 656 \mathrm{~N}(\mathrm{H} \alpha)$ and F658N ([NII]) narrow bandpass filters from the HST archive. Then, we obtained the wavelet decomposition of the $\mathrm{F} 656 \mathrm{~N}$ image using 6 wavelet scales (Figure 1). Scale $1\left(w_{1}\right)$ presents only one pixel-scale noise. The main bipolar structure, oriented roughly NS, appears in all other scales, $w_{2}-w_{6}$, with the strongest peak at scale $w_{4}$. The FLIERs can be seen as two well detached "blobs" in scales $w_{3}-w_{6}$.

It is noteworthy that scales $w_{3}$ and $w_{4}$ shown a faint secondary bipolar structure, close to the central part, that is aligned with the FLIERs and has never been reported before. This structure presents itself mainly in the form of an arc in the NE direction. It is detected only at the 2-sigma level, but can easily be identified as a continuous structure. A fainter and somewhat smaller symmetrical arc can be seen in the SW part of the image.

We have performed the same analysis for the F658N image, but no such structure was detected. The lack of detection does not necessarily imply that the structure is absent in $[\mathrm{NII}] 658 \mathrm{~nm}$. It could be attributed to the fact that it lies below the noise level in [NII], since its emission is approximately three times smaller than the $\mathrm{H} \alpha$ emission in this nebula (Acker et al. 1992). 


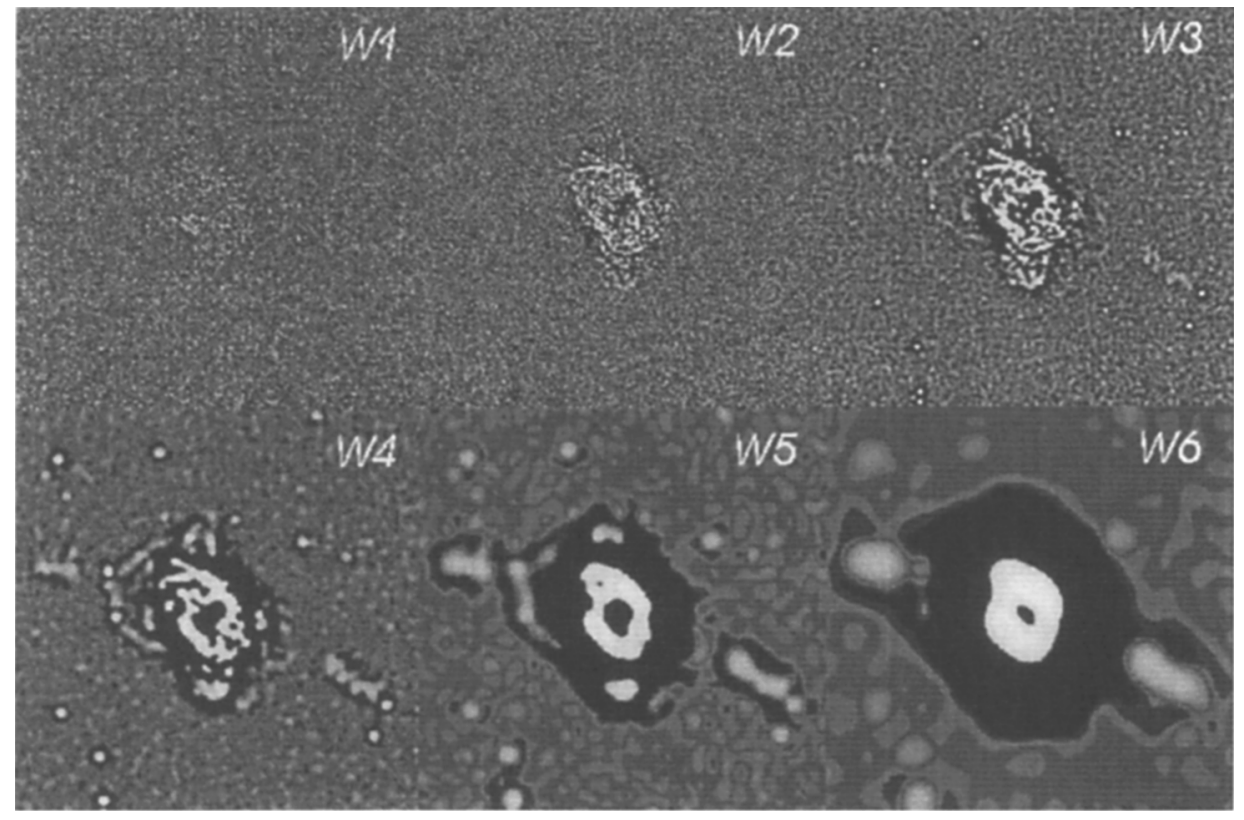

Figure 1. Images of the six first wavelet coefficients of Hubble 4.

\section{References}

Acker A., et al. 1992, Strasbourg-ESO Catalog of Planetary Nebulae, ESO Publ. Gonçalves, et al., 2001, ApJ, 547, 302

Hajian A., et al., 1997, AJ, 487, 304

López J., et al., 1997, ApJ, 485, 697

Starck, J. L., et al., 1998, Image and Data Analysis: The Multiscale Approach, Cambridge Un. Press 